\title{
Invariants of the group of a third order differential element.
}

\author{
Rodney Angottr (Buffalo U.S.A.)
}

Sammary. - In this note we discuss the invariant properties of the group of homographies which leave a third order differential element $E_{3}$ invariant.

1. - In [2] BoMpranr discussed the projective invariants of configurations associated with a third order curvilinear differential element $E_{3}$ in the ordinary projective space $P_{3}$; in particular, he showed that the configuration $C_{1}$ of the element $E_{3}$, a line $r$, and a point $P \notin r$ has a projective invariant and that the configuration $C_{2}$ of the element $E_{3}$ and two lines has two invariants. Furthermore, it is shown in [1] that the configuration $C_{2}$ has another invariant in the event that the two lines are incident and, in addition, that the invariants of $C_{\mathbf{2}}$ which Bompiant discusses are not geometric when the two lines are incident. The purpose of this note is to discuss the projective properties of the configuration consisting of the element $E_{3}$, a line $r$ and a plane $\pi \nsupseteq r$; in fact, we show that this configuration has a projective invariant and give an analytical formulation of this. We remark here that the configuration $C_{3}$ is not the dual configuration of $C_{1}$. In fact the dual configuration of $C_{2}$ would be a line, a plane and, using Bomprani's terminology, a particular psendo-element, i.e., an equivalence class of differential elements. For a precise definition of pseudo-element, see [3].

The author wishes to acknowledge here a communique from Professor Bompiani which was instrumental in the preparation of this manuscript.

In the following we will indicate points of $P_{3}$ by the ordered tetrad $(t, x, y, z)$ or, alternatively, by $x^{i}(i=0,1,2,3)$.

2. - Let $C$ be a curve passing regularly through the point $0(1,0,0,0)$ with tangent $y=z=0$ and osculating plane $z=0$. The equivalence class of curves (of at least class $C^{2}$ in a neighborhood of 0 ) which have a contact of order three with $O$ at 0 is called a third order curvilinear differential element $E_{3}$ with center 0 . This element can be represented analytically by the expansions $(t=1)$

$$
\begin{aligned}
& y=a x^{2}+a_{1} x^{3}+0\left(x^{4}\right) \\
& z=\quad b x^{3}+0\left(x^{4}\right)
\end{aligned}
$$


where the coefficients $a, a_{1}$, and $b$ are determined by $C$ and the symbol $O\left(x^{4}\right)$ indicates that the coefficients of the terms $>4$ are arbitrary.

By changing the plane $y=0$, we can always reduce the representation of the element $E_{3}$ to the form

$$
\begin{aligned}
& y=a x^{2}+0\left(x^{4}\right) \\
& z=b x^{3}+0\left(x^{4}\right)
\end{aligned}
$$

where $t=1, a \cdot b \neq 0$.

The group of homographies $G\left(E_{3}\right)$ which leave the expansions (2.1) invariant (or leave the element $E_{3}$ invariant) is represented by

where

$$
\begin{aligned}
& \lambda t^{\prime}=p_{1} t+p_{2} x+p_{3} y+p_{4} z \\
& \lambda x^{\prime}=q_{1} x+q_{2} y+q_{3} z \\
& \lambda y^{\prime}=p_{1}^{-1} q_{1}^{2} y+q_{4} z \\
& \lambda z^{\prime}=p_{1}^{-2} q_{1}^{3} z
\end{aligned}
$$

$$
q_{4}=a b^{-1} p_{1}^{-2} q_{1}\left(2 a p_{1} q_{2}-p_{2} q_{1}\right)
$$

Since each collineation of this group maps a generic line $r$, whose equations we can always write in the form

$$
\begin{gathered}
t-s_{2} y-s_{3} z=0 \\
x+h_{2} y+h_{3} z=0
\end{gathered}
$$

onto $t^{\prime}=x^{\prime}=0$, we can express these homographies more conveniently by the equations

where

$$
\begin{aligned}
& \lambda t^{\prime}=t-s_{1} x-\left(s_{2}+h_{2} s_{1}\right) y-\left(s_{3}+h_{3} s_{1}\right) z \\
& \lambda x^{\prime}=n\left(x+h_{2} y+h_{3} z\right) \\
& \lambda y^{\prime}=n^{2}(y+p z) \\
& \lambda z^{\prime}=n^{3} z
\end{aligned}
$$

$$
p=a b^{-1}\left(s_{1}+2 a h_{3}\right) \text {. }
$$

For a fixed line $r$, equation (2.3) represents a projectivity between the pencil of planes through the tangent (depending upon $p$ ) and the pencil of 
planes through $r$ (depending on $s_{1}$ ). The locus of line intersections of corresponding planes in this projectivity are generators of the quadric $Q_{r}$

$$
a z\left(t-s_{2} y-s_{3} z\right)+\left(b y+2 a^{2} h_{2} z\right)\left(x+h_{2} y+h_{3} z\right)=0
$$

By varying $r$, we obtain a linear system of quadrics of freedom three. This system is characterized geometrically in [2].

3. - Since $G\left(E_{3}\right)$ depends on six parameters, a generic line (which depends on four parameters) and a generic plane (which depends on three parameters) has an invariant under $\left.G_{(}^{\prime} E_{3}\right)$; in fact, we have the theorem:

Theorem 1. - Let $r$ be a generic line with equations (2.2) and $\pi$ be a plane, not passing through $r$, with plane-coordinates $u_{i}(i=0,1,2,3), u_{0} \neq 0$, then the function

$$
I(\pi, r)=\frac{\left[a u_{1} \pi_{1}+b u_{0}\left(\pi_{2}-k \pi_{1}\right)\right]^{2}}{u_{0} \pi_{1}^{3}}
$$

where

$$
\begin{aligned}
& \pi_{1}=u_{2}-u_{1} h_{2}+u_{0} s_{2} \\
& \pi_{2}=u_{3}-u_{1} h_{3}+u_{0} s_{3} \\
& k=2 a b^{-1} h_{2}
\end{aligned}
$$

is invariant under $G\left(E_{s}\right)$.

Proof: - Take a transformation $g \in G\left(E_{3}\right)$ which maps $r$ onto $r^{\prime}:[t=x=0]$. We have

$$
I\left(\pi^{\prime}, r^{\prime}\right)=\left[a \bar{u}_{1} \bar{u}_{2}+b \bar{u}_{0} \bar{u}_{3}\right]^{2} /\left(\bar{u}_{0} \bar{u}_{2}^{3}\right)
$$

where the $\bar{u}_{i}$ are the plane coordinates of $g \pi=\pi^{\prime}$. A calculation shows that for any $f \in G\left(E_{3}\right)$

$$
I(g \pi, g r)=I\left(\pi^{\prime}, r^{\prime}\right)=I\left(f \pi^{\prime}, f r^{\prime}\right)
$$

By choosing $f=g^{-1}$, we have

$$
I(g \pi, g r)=I(\pi, r) .
$$

Let $G(I(\pi, r))$ be the group of homographies which leave $I(\pi, r)$ inva. riant. Obviously $G\left(E_{3}\right) \subset G(I(\pi, r))$. In the following we show that $G(I(\pi, r)) \subset$ $\subset G\left(E_{3}\right)$ and, consequently, $G(I(\pi, r))=G\left(E_{3}\right)$. 
Lemma. - A homography $f$ which leaves the line $r^{\prime}:[t=x=0]$ invariant and has the property that $I\left(\pi, r^{\prime}\right)=I\left(f \pi, r^{\prime}\right)$, identically with respect to $\pi$, belongs to $G\left(E_{3}\right)$.

Proof. - If the homography $f$ has the form $x^{i^{\prime}}=a_{i j} x^{j},(i, j=0,1,2,3)$, then $a_{i j}=0$, for $i \neq j$, and

$$
\begin{aligned}
& a_{33}=a_{11}^{-1} a_{22}^{2} \\
& a_{44}=a_{11}^{-2} a_{22}^{3}
\end{aligned}
$$

which imply that $f \in G\left(E_{3}\right)$.

Now take a homography $h \in G(I(\pi, r))$. The homography $h$ has the property

$$
I(h \pi, h r)=I(\pi, r)
$$

for generic $\pi$ and $r$. There obviously exist homographies $g_{1}, g_{2} \in G\left(E_{8}\right)$ which map $r$ and $h r$ onto $r^{\prime}:[t=x=0]$, respectively. Since $g_{1}, g_{2}$ leave (3.1) inva. riant, we have

$$
I\left(g_{1} \pi, g_{1} r\right)=I(\pi, r)=I(h \pi, h r)=I\left(g_{2} h \pi, g_{2} h r\right)
$$

The homography $f=g_{2} h g_{1}^{-1}$ maps $g_{1} r$ onto $g_{2} h r=r^{\prime}$ and leaves (3.1) invariant; therefore, by the Lemma it belongs to $G\left(E_{3}\right)$. The equality $h=g_{2}^{-1} f g_{1}$ implies that $h \in G\left(E_{3}\right)$. We have proved the following theorem:

Theorem 2. - A homography of $P_{3}$ which leaves $I(\pi, r)$ invariant is a transformation of the group $G\left(E_{3}\right)$.

CoRoldary. - The invariant 3.1 ) of a plane and line completely characterizes the group $G\left(E_{3}\right)$.

We propose to show that the invariant $I(\pi, r)$ is transitive with respect to $G\left(E_{3}\right)$, i.e., there exists a transformation which maps a given plane-line pair $\pi_{1}, r_{1}$ onto any other plane-line pair $\pi_{2}, r_{2}$ for which $I\left(\pi_{1}, r_{1}\right)=I\left(\pi_{2}, r_{2}\right)$. Clearly there exist transformations, say $f$ and $g$, of $G\left(E_{3}\right)$ which map $r_{1}, r_{2}$ onto $r^{\prime}:[t=x=0]$, respectively. Since $f, g \in G\left(E_{*}\right)$, we have

$$
I\left(\pi_{1}, r_{1}\right)=I\left(f \pi_{1}, f r_{1}\right)=I\left(g \pi_{2}, g r_{2}\right)=I\left(\pi_{2}, r_{2}\right)
$$

There exists, however, a unique transformation $h \in G\left(E_{3}\right)$ which leaves $r^{\prime}$ invariant and is such that $h f \pi_{1}=g \pi_{2}$. Therefore, the composition $g^{-1} h f$ maps 
$r_{1}$ onto $r_{2}, \pi_{1}$ onto $\pi_{2}$ and has the property that

$$
I\left(\pi_{1}, r_{1}\right)=I\left(g^{-1} h f \pi_{1}, g^{-1} h f r_{1}\right)=I\left(\pi_{2}, r_{2}\right) .
$$

Corollary. - The invariant $I(\pi, r)$ is the only (independent) invariant of a plane and line under transformations of $G\left(E_{3}\right)$.

4. - In order to give a geometrical construction of the invariant $I(\pi, r)$, consider the envelopes of the quadrics which contain the element $E_{3}$, the line $r$, and are tangent to the plane $\pi$. The residual intersection of this system is a cubic developable on which there are four well-determined planes; namely, the plane $\pi$, the osculating plane $z=0$ and the two planes of the developable intersecting in the line $r$. It is only a matter of calculation to verify that the cross ratio of these four planes is a rational function of $I(\pi, r)$.

This invariant can be interpreted more directly as the cross ratio of four points on the unique skew cubic $C^{3}$ which contains the element $E_{3}$ and is tangent to the line $r$ at the point $P=r \cap \pi$. In this case, the four points are the center 0 of the element $E_{3}$, the point $P$, and the two additional points, say $Q, R$, in which the plane $\pi$ euts $C^{3}$. This construction gives rise to several other equivalent interpretations of the invariant $I(\pi, r)$.

5. - If we fix the line $r$, the set of planes for which $I(\pi, r)=0$ is given by

$$
a u_{1}\left(u_{2}-u_{1} h_{2}+u_{0} s_{2}\right)+b u_{0}\left[u_{3}-k u_{2}-\left(h_{3}-h_{2} k\right) u_{1}+\left(s_{3}-k s_{2}\right) u_{0}\right]=0
$$

where $k=2 a b^{-1} h_{2}$. This locus is the envelope of the quadric $Q_{r}$ obtained from the projectivity (2.3). More generally, the planes for which $l(\pi, r)$ is constant, $r$ fixed, belong to the fourth class envelope in the pencil determined by the envelope of the quadric $Q_{r}$, counted twice, and the envelope composed of the star of planes through the center 0 of the element $E_{3}$ and the pencil of planes with axis $r$, counted three times.

\section{REFERENCES}

[1] R. AngotT, The Projective Invariants of Two Lines and a Third Order Differential Element, «Rend. Somin. Padovas, 35 (1965).

[2] E. Bompranr. Configurations associées à un élément curviligne du troisieme ordre dans l'espace projectif, «Journal des Math. pure et appl.», 41 (1962).

[3] E. Bompiani, Nuovi Enti Geometrici: Pseudoelementi Differenziali, *Sem. Mat, e Fisico* 33 (1963). 